\title{
Systematic Current Control Strategy with Pole Assignment for Grid-Connected LCL-Filtered Inverters
}

\author{
Jinming $\mathrm{Xu}^{\dagger}$, Shaojun $\mathrm{Xie}^{*}$, and Ting Tang ${ }^{*}$ \\ ${ }^{\dagger *}$ College of Automation Engineering, Nanjing University of Aeronautics and Astronautics, Nanjing, P. R. China
}

\begin{abstract}
For grid-connected LCL-filtered inverters, resonance yields instability and low bandwidth. As a result, careful designs are required. This paper presents a systematic current control structure, where pole assignment consisting of one or more feedbacks is the inner loop, and the outer loop is the direct grid current control. Several other issues are discussed, such as the inner-loop feedback choices, pole-assignment algorithms, robustness and harmonic rejection. Generally, this kind of strategy has three different types according to the inner-loop feedback choices. Among them, a novel pole-assignment algorithm has been proposed, where the inner control maintains four freely-assigned poles which are just two pairs of conjugated poles located at the fundamental and resonance frequencies separately. It has been found that with the different types, the steady-state and dynamic performances are quite different. Finally, simulations and experiments have been provided to verify the control and design of the proposed methods.
\end{abstract}

Key words: Active damping, Grid-connected inverter, LCL filters, Pole assignment, Systematic control

\section{INTRODUCTION}

The grid-connected inverter is one of the key technologies for distributed power generation systems (DPGSs) based on renewable energy sources. The functions of the grid-side converter consist of active and reactive power control, dc-link voltage control, attainment of a high quality injected current and phase locked loop (PLL) for grid synchronization [1]. In particular, strict standards in respect to current quality are issued in various countries, including Total Harmonic Distortion (THD) and individual harmonic distortions. To effectively attenuate current harmonics around the switching frequency, LCL filters are preferred in pulse width modulated (PWM) voltage-source converters [2], [3]. Compared with L filters, LCL-type filters offer better performance. However, the resonance caused by the three-order filter puts forward a higher requirement for the overall system control.

Firstly, since the impedance of the filters at the resonance

Manuscript received Aug. 26, 2012; revised Jan. 23, 2013

Recommended for publication by Associate Editor Kyo-Beum Lee.

'Corresponding Author: xjinming01@163.com

Tel.: +86 13913391056, Fax: +86 25-84892361, Nanjing University of Aeronautics and Astronautics

"College of Automation Engineering, Nanjing University of Aeronautics and Astronautics, P. R. China frequency is rather low, the harmonics around this frequency are extremely considerable. In [2], [3], a passive resistor is inserted in series with a capacitor, and the resonance is highly attenuated. However, the power losses and the weakened rejection of high-frequency harmonics are undesirable. Alternatively, many researchers have used additional control to realize the same damping purpose, named active damping (AD). Compared with passive methods, $\mathrm{AD}$ methods do not produce the mentioned demerits. Hence they have been widely considered in recent years. AD based on attaching filters on the forward path to attract the resonance poles to the stable region can be seen in [4]-[6]. This does not require any additional sensors. However, it has been shown that the additional pole-zero filters should be placed near the resonance poles, so that its effectiveness and robustness are highly related to the model accuracy [5]. In contrast, another kind of $\mathrm{AD}$, which uses feedback control, can guarantee better robustness. According to the number of additional feedbacks, the strategies can be sorted: AD based on a single state feedback such as the capacitor current [7]-[10] or capacitor voltage [10]-[12], and multi state feedback [13]. These AD methods have one thing in common. They are all treated as an inner-loop control to move the natural resonance poles toward the left complex plane. With the inner $\mathrm{AD}$ 
control, the original LCL system is transformed into a damped one, and then the follow-up design of the current loop can be performed. The drawback of these AD methods is the requirement of many sensors; especially the AD in [13] which requires three sensors. Certainly, there is one possible solution to adopting the observation control [12]. However, the observation still requires system model accuracy, and its effectiveness and robustness still need be verified.

Secondly, even when the original system has been well damped with $\mathrm{AD}$, instability would still occur if the filter parameters vary or the design is not proper. The authors of [10] have indicated that the feedback control of capacitor current or voltage can not result in effective resonance damping in different filter parameters cases, for example variations of the capacitance value. This was actually due to the large variation of the resonance frequency. In this case, greatly reducing the system bandwidth may make the system stable. However, this results in poor transient response and harmonics rejection. This means, that $\mathrm{AD}$ using a single state feedback is not suitable because some system feedback information was missed. This has been pointed out in [8] where the authors indicated that the system pole-zero placement design met constrains. However, the complete state feedback adopted in [13], which may fix this, is also not desirable because of its drawback. The inner loop control and design methods for the LCL system still require further and systematic analysis.

Thirdly, whether the fundamental and harmonics current can track the reference accurately is also in the stage of exploration. In the rotating frame, the ac grid current and voltage waveforms are transformed into dc components. A good steady-state response can be easily achieved with a simple PI regulator. However, in the stationary frame, it is difficult to guarantee an extremely small steady-state error with a PI controller [1]. During last decade, researchers have been focusing on the study of current regulators. Proportional and resonant (PR) controllers, which achieve an extremely high gain at the fundamental frequency, can be seen in [14]-[16]. However, PR controllers are so sensitive to parameter variations such as grid frequency deviation [14] that a slight damping is required for the purpose of robust performance. As for the harmonics compensations in both frames, multi-resonant controllers have been adopted in [14], [15]. In addition, a full feedforward method consisting of proportional and derivative feedforward functions has been proposed in [9] to suppress grid distortions. With the mentioned complex methods, precise tracking can be achieved. However, so far, not enough attention has been paid to inner-loop control, since it also affects the tracking performance.

In view of the aforementioned issues, there is a strong demand to fully recognize the control of grid-connected LCL-filtered inverters. The contribution of this paper is to

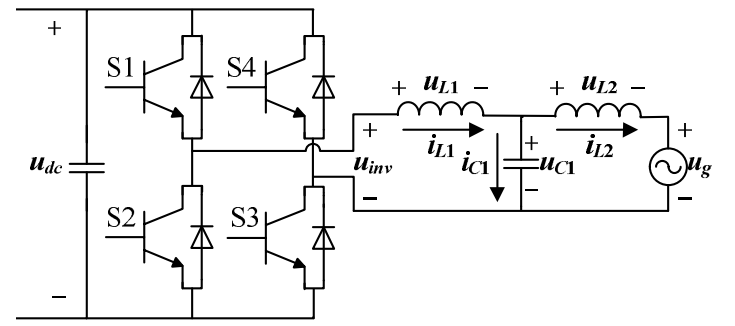

Fig. 1. Single-phase grid-connected LCL-filtered inverter.

promote the analysis. At first, a systematic control structure for LCL-filtered inverters is introduced, where the pole assignment control consisting of one or more feedbacks is treated as the inner-loop control and the outer loop is the direct current control. The proposed structure covers most of the aforementioned feedback-based AD methods. Thus it provides a way to systematically study the design and performance of such methods. Then, comprehensive studies of the pole assignment control are provided. Furthermore, the design methods, control robustness and harmonics rejection are discussed. Guidelines and some additional recommendations are also presented. Finally, simulations and experiments verify the proposed systematic control.

\section{SYSTEM DESCRIPTIONS}

Generally, a three-phase system can be equivalent to two single-phase systems through a frame transformation [1]. The current control for a single-phase inverter is discussed. Figure 1 shows the LCL-filtered inverter structure, which consists of the inverter-side inductance $L_{1}$, filter capacitance $C_{1}$, grid-side inductance $L_{2}$, dc-link voltage $u_{d c}$, inverter output voltage $u_{i n v}$, grid voltage $u_{g}$ (sampled for the feedforward and the PLL), inverter-side inductor voltage $u_{L 1}$, capacitor voltage $u_{C 1}$, grid-side inductor voltage $u_{L 2}$, inverter current $i_{L 1}$, capacitor current $i_{C 1}$ and grid current $i_{L 2}$. The relation between $u_{i n v}$ and $i_{L 2}$ is shown in (1), where $r_{1}$ and $r_{2}$ represent the equivalent series resistances (ESRs) of the two inductors. As indicated in Fig. 2, the high-frequency harmonics can be highly attenuated. However, the current is easily resonated because of the peak at the natural resonance frequency $\left(\omega_{\text {res }}\right.$, expressed in (2)).

$$
\begin{gathered}
G_{u_{i n v}}^{i_{L 2}}(s)=1 /\left[L_{1} L_{2} C_{1} s^{3}+\left(r_{2} L_{1}+r_{1} L_{2}\right) C_{1} s^{2}\right. \\
\left.+\left(L_{1}+L_{2}+r_{1} r_{2} C_{1}\right) s+r_{1}+r_{2}\right] \\
\omega_{\text {res }}=\sqrt{\frac{L_{1}+L_{2}}{L_{1} L_{2} C_{1}}}
\end{gathered}
$$

The simplified single grid current control is expressed in Fig. 3, where $k_{g}$ is the coefficient of the grid feedforward function; $G_{c}(s)$ is the outer-loop regulator which performs precise tracking; $u$ is the output of the current regulator; and $i_{\text {ref }}$ is the reference current. As can be seen in Fig. 2, since the phase curve rapidly varies from $-90^{\circ}$ to $-270^{\circ}$ around the 


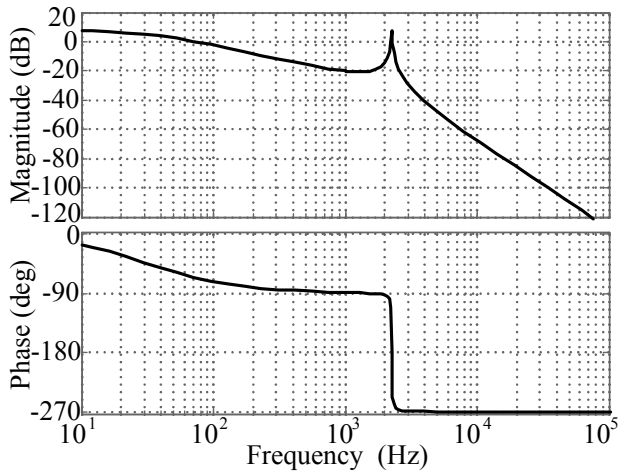

Fig. 2. Grid current amplitude and phase characteristics.

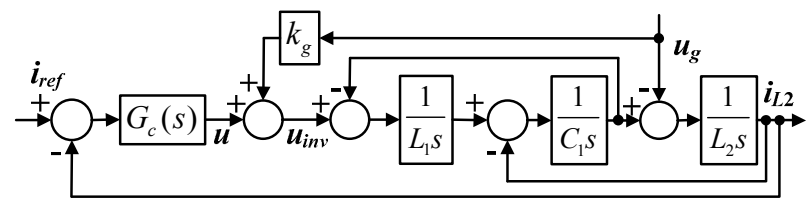

Fig. 3. Single grid current control structure.

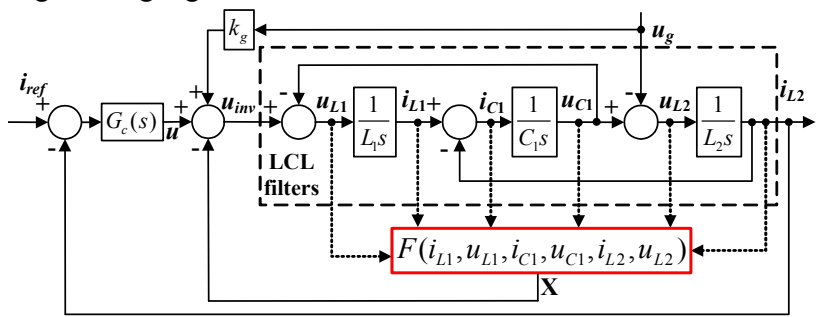

Fig. 4. Systematical control strategy for grid-connected LCL-filtered inverters.

resonance frequency, according to the stability criterion the system is easily resonated when the loop is closed. The current regulator should guarantee that the resonance amplitude is attenuated below $0 \mathrm{~dB}$. Besides, in order to guarantee proper amplitude and phase margins, the regulator design is extremely restricted and the system performance is poor.

Thus the peak needs to be attenuated. One effective way to achieve this is to introduce an inner feedback control. As can be seen in Fig. 1, at least six variables including the currents and voltages of the three filter components can be fed back with the proportional (P), integral (I) and derivative (D) functions. In this paper, a systematical control structure for LCL-filtered inverters has been proposed, as shown in Fig. 4. The overall control has been treated as a dual-loop control. The combined feedback $X$ of the six variables realizes the pole assignment, which forms the inner-loop control to improve the system performance. In addition, the $F$ function denotes the P, I and D functions of the currents and voltages. Regulator $G_{c}(\mathrm{~s})$ and grid feedforward $k_{g}$ perform precise tracking of the fundamental and harmonics currents, which are treated as the outer-loop control. Although the ESRs can provide some inherent damping of the LCL resonance, without additional passive or active damping the system bandwidth is too low to promise good transient performance or high attenuation of the low-order current harmonics [14]. Since a system without ESRs represents the worst case for the design [17], the ESRs have been ignored in this paper.

\section{DISCUSSIONS OF THE SYSTEM CONTROL}

Straightforward design procedures are proposed in [8] and [13] where the overall close-loop transfer function is calculated, and then the pole-zero assignment is used. It cannot be applied anywhere else because systems with different controls may have a different number of freely-placed poles and zeros. In addition, it is hard to obtain the overall transfer function. Thus in this paper the design of the systematical control is equivalently seen as a typical dual-loop structure.

\section{A. Pole Assignment Control}

The inner-loop control is a linear combination of the state variable feedbacks in Fig. 4. In total, there are $18(6 \times 3)$ kinds of feedbacks. Among the current and voltage variables, $u_{L 1}$ is the difference between $u_{i n v}$ and $u_{C 1}$. It is difficult to sample this PWM-shape voltage. Even if the voltage is effectively sampled, the PWM function will be ineffective if the high switching harmonics-featured signal is directly used for the feedback control. Besides, it is hard to accurately obtain the necessary control information from this sampled signal. Thus it is not suitable to perform proportional or derivative feedback of $u_{L 1}$. The integral function has the characteristic of filtering the voltage signal, and may be implemented. The derivative feedback of $i_{L 1}$ or $i_{C 1}$ meets the same constraint due to the large amount of high-frequency harmonics. Hence the number of available feedbacks is reduced to 14 . Besides, seen from the forward path from the reference to the grid current and taking the grid as a disturbance, the capacitor voltage and the grid-side inductor voltage are the same. Thus they have the same impact on system stability. However, they have a different impact on harmonics rejection because the main harmonics source is the grid voltage which is included in the capacitor voltage. In this section, $u_{C 1}$ and $u_{L 2}$ are treated the same. Then, the combination can be expressed as:

$$
\begin{aligned}
\mathrm{X} & =i_{L 1} \cdot\left(x_{P}+\frac{x_{I}}{s}\right)+u_{L 1} \cdot \frac{y_{I}}{s}+i_{C 1} \cdot\left(z_{P}+\frac{z_{I}}{s}\right)+\left(u_{C 1}\right. \\
& \left.+u_{L 2}\right) \cdot\left(p_{P}+\frac{p_{I}}{s}+p_{D} s\right)+i_{L 2} \cdot\left(q_{P}+\frac{q_{I}}{s}+q_{D} s\right)
\end{aligned}
$$

where, $x, y, z, p$ and $q$ represent the feedback factors of $i_{L 1}$, $u_{L 1}, i_{C 1}, u_{C 1}\left(u_{L 2}\right)$ and $i_{L 2}$, respectively, and the subscripts ' $P$ ', ' $I$ ' or ' $D$ ' denote $\mathrm{P}$, I or $\mathrm{D}$ functions. The adoption of all of the feedbacks is unreasonable because this results in too many sensors. It is required to find a proper method including feedback combination (with the lowest number of feedbacks or sensors) and parameters design.

As long as the transfer function reveals all of the 
information on the system dynamic, the way to fix the problem is to derive it first. A simplified method based on Mason's gain formula [18]-[20] is used:

$$
G(s)=\frac{1}{1-\sum L_{a}+\sum L_{b} L_{c}} \sum_{i=1}^{n} d_{i} \cdot\left(1-\Delta_{i}\right)
$$

where, $d_{i}$ is the product of all the transfer functions along the $i$-th forward path from $u$ to $i_{L 2} ; \Delta_{i}$ is the sum of the gains of the loops which have no contact with the $i$-th forward path; the summation is over all of the $n$ forward paths; $\sum L_{a}$ equals to the sum of all of the individual loop gains; and $\sum L_{b} L_{c}$ is the sum of the products of the gains of all possible two loops that do not touch each other.

Here, as seen from Fig. 4, there is only one forward path from $u$ to $i_{L 2}$. The detailed ones are given as:

$$
\left\{\begin{array}{l}
n=1 \quad d_{1}=\frac{1}{L_{1} s} \cdot \frac{1}{C_{1} s} \cdot \frac{1}{L_{2} s} \Delta_{1}=0 \\
\sum L_{a}=-\left[\begin{array}{l}
\left.\frac{1}{L_{1} s_{1}} \cdot \frac{1}{C_{1} s}+\frac{1}{C_{1} s} \cdot \frac{1}{L_{2} s}+\frac{y_{I}}{s}+\frac{1}{L_{1} s} \cdot\left(x_{P}+\frac{x_{I}}{s}\right)+\frac{1}{L_{1} s} \cdot\left(z_{P}+\frac{z_{I}}{s}\right)+\right] \\
\frac{1}{L_{1} s} \cdot \frac{1}{C_{1} s} \cdot\left(p_{P}+\frac{p_{I}}{s}+p_{D} s\right)+\frac{1}{L_{1} s} \cdot \frac{1}{C_{1} s} \cdot \frac{1}{L_{2} s} \cdot\left(q_{P}+\frac{q_{I}}{s}+q_{D} s\right)
\end{array}\right] \\
\sum L_{b} L_{c}=-\left(\frac{y_{I}}{s}\right) \cdot\left(-\frac{1}{C_{1} s} \cdot \frac{1}{L_{2} s}\right)-\left(x_{P}+\frac{x_{I}}{s}\right) \cdot\left(\frac{1}{L_{1} s_{1}}\right) \cdot\left(-\frac{1}{C_{1} s} \cdot \frac{1}{L_{2} s}\right)
\end{array}\right.
$$

Substituting (5) into (4), the transfer function can then be expressed as:

$$
\begin{aligned}
G_{u}^{i_{L 2}}(s) & =\frac{1}{b_{0} s^{3}+b_{1} s^{2}+b_{2} s^{1}+b_{3} s^{0}+b_{4} s^{-1}} \\
b_{0} & =L_{1} L_{2} C_{1} \\
b_{1} & =L_{2} C_{1} x_{P}+L_{1} L_{2} C_{1} y_{I}+L_{2} C_{1} z_{P}+L_{2} p_{D} \\
b_{2} & =L_{2} C_{1} x_{I}+L_{2} C_{1} z_{I}+L_{2} p_{P}+q_{D}+L_{1}+L_{2} \\
b_{3} & =x_{P}+L_{1} y_{I}+L_{2} p_{I}+q_{P} \\
b_{4} & =x_{I}+q_{I}
\end{aligned}
$$

It is clear that the coefficients, except $b_{0}$, can be assigned freely. Consequently, the original resonated system can be controlled to be a well-damped one. In other words, the resonance damping can be realized perfectly. Obviously, for the purpose of fully configuring the characteristic equation, it is unnecessary to feedback all of the state variables. The feedback choice only needs to realize the assignment of every coefficient in (6).

As mentioned before, the peak in Fig. 2 yields the demerits of resonance and poor performance. Seen from (6), with the feedback control, the coefficient $b_{1}$ is assigned so that the peak suppression is guaranteed. The assignment of $b_{2}$ mainly shifts the resonance frequency. With the adoption of $b_{3}$ and $b_{4}$, one or more configurable poles can be obtained. These result in different parameter designs. In summary, for the purpose of inner-loop stability, the coefficient $b_{1}$ must not be zero. However, the coefficients $b_{3}$ and $b_{4}$ can be zero. Generally, the characteristic equation can be configured into three different types.

$$
\begin{array}{ll}
\text { Type I: } & D_{1}(s)=b_{0} s^{3}+b_{1} s^{2}+b_{2} s \\
\text { Type II: } & D_{2}(s)=b_{0} s^{3}+b_{1} s^{2}+b_{2} s+b_{3} \\
\text { Type III: } & D_{3}(s)=s^{-1}\left(b_{0} s^{4}+b_{1} s^{3}+b_{2} s^{2}+b_{3} s+b_{4}\right)
\end{array}
$$

For the different types, the pole locations are quite different.

Type I:

The desired characteristic equation can be expressed as:

$$
D_{1}(s)=b_{0} s\left(s^{2}+2 \zeta \omega_{n} s+\omega_{n}^{2}\right)
$$

where, $\omega_{n}$ is the frequency of the conjugated poles and $\zeta$ is the damping factor, which generally equals $0.6 \sim 0.8$ when considering the resonance damping and phase margin. By comparing (7) with (10), the parameters of the combined feedback can then be obtained. One thing to be noted, the assignment of $b_{2}$ has been realized with the combined feedback control. Thus the frequency $\omega_{n}$ can be designed independently according to the requirements of system stability and dynamics, and will be discussed in the next section.

Type II:

The only difference between (8) and (7) is the assignment of $b_{3}$. This means, the real pole in (7) is located at zero while in (8) is located on the negative real axis of the $s$-plane. The equation is expressed as:

$$
D_{2}(s)=b_{0}\left(s+m \zeta \omega_{n}\right)\left(s^{2}+2 \zeta \omega_{n} s+\omega_{n}^{2}\right)
$$

where, $m$ is a positive number reflecting the real pole position. In general, $m$ can be $4 \sim 6$. In this case, the frequency of the real pole is larger than $\omega_{n}$ (for instance, if $\zeta$ is 0.707 the ratio is about 3). Consequently, the conjugated poles dominate the system dynamics, and the system is similar to a second-order system [20]. Therefore, the design can be degraded. Like Type I, the parameters of the combined feedback can then be obtained.

Type III:

When the characteristic equation is configured into (9), four freely-placed poles are yielded. However, a zero located at zero is introduced, which results in a decrease in the amplitude-frequency response in the low-frequency region. This configuration does not seem to favor the elimination of steady-state errors when the system is closed. However, besides the two conjugated poles located at $\omega_{n}$, the two remaining poles can also be conjugated poles located at a specific frequency. Typically, for control in the stationary frame it can be the fundamental frequency $\omega_{0}$. Consequently, if the damping factor $\zeta_{0}$ is rather small, the control performs similar to a PR controller [15] without the adoption of PR control. The desired equation is expressed as:

$$
D_{3}(s)=b_{0} s^{-1}\left(s^{2}+2 \zeta_{0} \omega_{0} s+\omega_{0}^{2}\right)\left(s^{2}+2 \zeta \omega_{n} s+\omega_{n}^{2}\right)
$$

The parameters of the combined feedback can be easily obtained by comparing (9) with (12). 
TABLE I

PARAMETERS OF GRID-CONNECTED LCL INVERTER

\begin{tabular}{c|c|c}
\hline Symbol & Description & Value \\
\hline$U_{g}$ & Grid voltage (RMS) & $220 \mathrm{~V}$ \\
$f_{0}$ & Grid frequency & $50 \mathrm{~Hz}$ \\
$U_{D C}$ & DC-link voltage & $400 \mathrm{~V}$ \\
$P$ & Rated output power & $3 \mathrm{~kW}$ \\
$f_{s}$ & Switching frequency & $10 \mathrm{kHz}$ \\
$L_{1}$ & Inverter-side inductance & $1 \mathrm{mH}$ \\
$L_{2}$ & Grid-side inductance & $1 \mathrm{mH}$ \\
$C_{1}$ & Filter capacitance & $10 \mu \mathrm{F}$ \\
\hline
\end{tabular}

\section{B. Overall Design Discussions}

The above section only presents the general methods for choosing feedbacks and obtaining parameters for the inner pole-assignment control. However, discussion on the detailed value of parameters is still open. It is well known that if the outer loop is closed, the poles of the overall system drift away from the ones assigned by the inner-loop control. Then, there exists a problem: the coordination between the inner and outer loops. Based on a study of system dynamics, detailed discussions are issued below. For analysis, the system parameters are shown in Table I.

For the dual-loop design, one issue is whether the overall system can perform accurate reference tracking. It can be seen in many studies that precise tracking is mainly determined by the outer-loop regulator. However, for purposes of control coordination and simplification, the inner loop should be beneficial for the tracking performance. Figure 5 shows the inner-loop control performance of the three configuration types with the parameters shown in Table I and (13). For analysis, the value of $\omega_{n}$ is given first. Its design guideline will be discussed later. Besides, $\zeta_{0}$ determines the bandwidth of the fundamental resonant control. In practice, it can be designed as illustrated in [14]. The value in (13) is just for analysis.

$$
\left\{\begin{array}{l}
\zeta=0.6, \omega_{n}=\omega_{\text {res }}, m=4 \\
\zeta_{0}=0, \omega_{0}=2 \pi f_{0}
\end{array}\right.
$$

It can be seen from Fig. 5 that with the Type I configuration, the system has infinite amplitude-frequency response of the dc component, while the gain of the fundamental frequency is relatively low. With the Type II configuration, the system has a poor amplitude and frequency response for both the $\mathrm{dc}$ and fundamental frequency component. However, systems with the Type III algorithm can obtain an extremely high gain at the fundamental frequency. It is well known that the higher the gain of the open-loop response the better the close-loop response. In conclusion, Type I and Type III algorithms are better than Type II.

Another important issue is whether the overall system can guarantee a suitable bandwidth and effective resonance

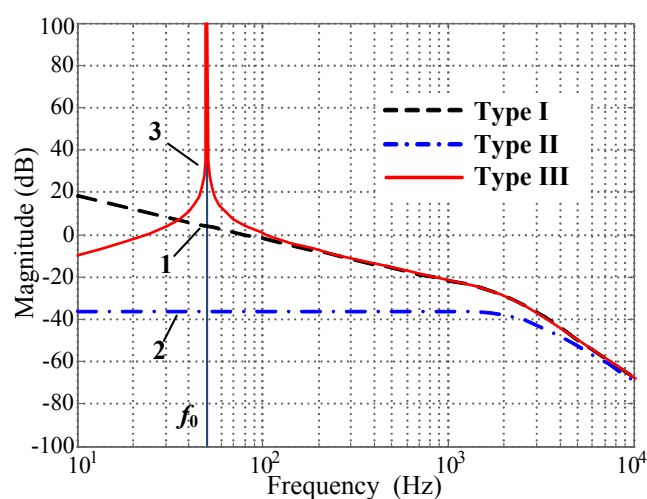

Fig. 5. Comparisons of different inner-loop control types.
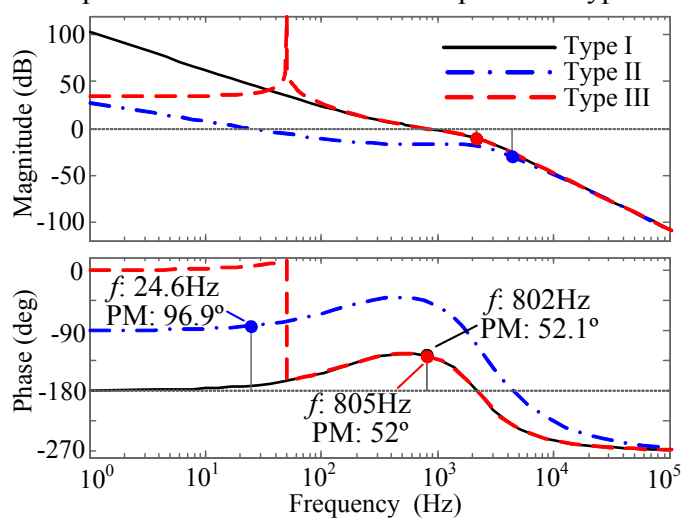

Fig. 6. Open-loop characteristics with different algorithms.

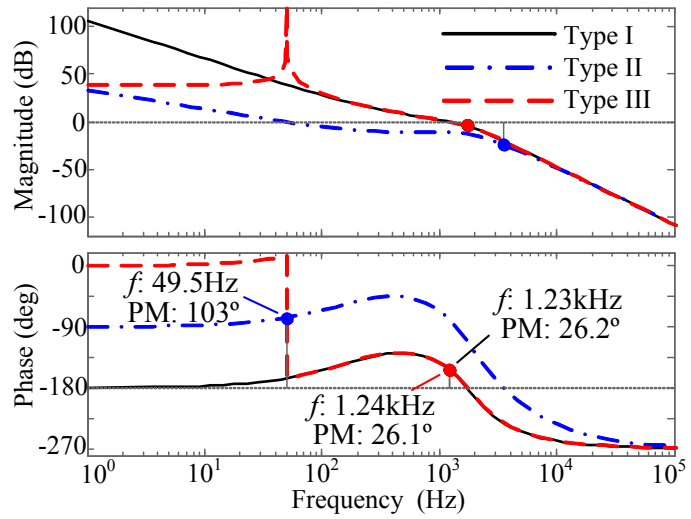

Fig. 7. Open-loop characteristics when $\omega_{n}$ becomes smaller.

damping. For instance, an outer-loop PI regulator is chosen with the parameters expressed in (14). It is indicated in [5] that an L-filtered $\left(L=L_{1}+L_{2}\right)$ inverter with this optimal PI controller can realize a considerably high bandwidth (nearly $1 / 5$ of the switching frequency $f_{s}$ ). Because the LCL-filtered inverter has a similar low-frequency response, this controller is also preferred. In this paper, $a_{i}$ is chosen to be 3 .

$$
\begin{aligned}
& G_{c}(s)=k_{p} \cdot \frac{T_{i} s+1}{T_{i} s} \\
& k_{p}=\frac{1}{2}\left(L_{1}+L_{2}\right) f_{s} \quad T_{i}=\frac{a_{i}^{2}}{f_{s}}
\end{aligned}
$$

Then the open-loop transfer function is (where, the 
subscript ' $O$ ' denotes the open loop):

$$
G_{i_{\text {ref_o }} i_{L 2}}^{i_{2}}(s)=k_{p} \cdot \frac{T_{i} s+1}{T_{i} s} \cdot G_{u}^{i_{L 2}}(s)
$$

Figure 6 expresses the open-loop characteristics with the parameters shown in (13) and (14), where PM represents the phase margin. These plots also indicate the demerit of the steady-state performance of Type II. Besides, although the resonance rejection of Type II is the best, the PM (about $100^{\circ}$ ) and the low cut-off frequency (about $25 \mathrm{~Hz}$ ) result in an extremely poor dynamic response. Thus $k_{p}$ needs to be increased a lot. However, Type II cannot perform similar to Type I or III because the gain margin (GM) is rapidly decreased or even below zero if $k_{p}$ is greatly increased (and in practice it is difficult to greatly increase $k_{p}$ with either digital or analog control; and the following studies still use the PI in (14)). In contrast, Types I and III guarantee a suitable PM and GM. The close-loop bandwidth is about $1.8 \mathrm{kHz}$. It is to be noted that $k_{p}$ can still be increased slightly.

In addition, it can be seen from Fig. 6 that the crossovers (in phase curves) of Types I and III both occur at around $\omega_{n}$; and that of Type II occurs at a frequency higher than $\omega_{n}$ due to the parameter $m$. According to the Nyquist stability criterion, once a PI controller makes the cut-off frequency close to or higher than the cross-over frequency, the stability margins decrease rapidly and instability can occur. Thus it can be deduced that the system bandwidth which is related to $k_{p}$ is limited if the frequency $\omega_{n}$ is low. This is further verified in Fig. 7, where the parameters are the same as Fig. 6 except for $\omega_{n}$ which is $4 / 5$ of that shown in (13). Obviously, the PMs of Types I and III are only half of that shown in Fig. 6, and the GMs are only $4.29 \mathrm{~dB}$. It is to be noted that if the value of $\omega_{n}$ is even smaller, the system cannot be stable. In this case, it is necessary to decrease $k_{p}$; and system will perform poorly.

Thus for the purposes of avoiding interaction conflict and simplifying designs, it is recommended that Type II is not used, and that $\omega_{n}$ should be higher than the desired bandwidth by a suitable margin. For example, if the desired bandwidth is $1 / 5$ of $f_{s}$, it is suggested that $\omega_{n}$ be:

$$
\omega_{n}>0.4 \pi f_{s}
$$

In practice, if the natural frequency $\omega_{\text {res }}$ satisfies (16), the assigned frequency $\omega_{n}$ can equal to $\omega_{\text {res }}$ for simplification; otherwise, $\omega_{n}$ must be rearranged according to (16). However, $\omega_{n}$ is not supposed to be too high because of the PWM model accuracy. This is the reason why that value in (13) is chosen.

In addition, a digital control delay is always in existence. In view of a simple and unified design, it is not taken into account in the above sections. Having considered the loop delay in the forward path (one-sample delay as indicated in [5], [13]), the performances of the three control types have been studied. Compared with Fig. 6, the delay actually decreases the PM depending on the control/sample frequency. Take Type III control for instance: if the control frequency
TABLE II

Characteristics When Filter Parameters Vary

\begin{tabular}{cc|ccc}
\hline \multicolumn{2}{c}{ Cases } & Type I & Type II & Type III \\
\hline \multirow{2}{*}{$\mathrm{GF}$} & $L_{1}$ & $33 \sim 37.2$ & -5.94 & $45.8 \sim 50$ \\
$(\mathrm{~dB})$ & $C_{1}$ & 34.7 & -5.94 & $50.2 \sim 51$ \\
& $L_{2}$ & $32.9 \sim 37.4$ & -5.95 & $45.2 \sim 50.7$ \\
\hline \multirow{3}{*}{$\mathrm{PM}$} & $L_{1}$ & $44.2^{\circ} \sim 58.2^{\mathrm{o}}$ & $96.8^{\mathrm{o}}$ & $44.1^{\circ} \sim 58.2^{\mathrm{o}}$ \\
& $C_{1}$ & $43.0^{\circ} \sim 65.2^{\mathrm{o}}$ & $96.9^{\mathrm{o}}$ & $42.9^{\circ} \sim 65.2^{\circ}$ \\
& $L_{2}$ & $51.5^{\mathrm{o}} \sim 56.3^{\mathrm{o}}$ & $96.5^{\mathrm{o}}$ & $51.5^{\circ} \sim 56.2^{\circ}$ \\
\hline \multirow{2}{*}{$\mathrm{GM}$} & $L_{1}$ & $8.9 \sim 13.8$ & $26.5 \sim 36.5$ & $8.89 \sim 13.8$ \\
$(\mathrm{~dB})$ & $C_{1}$ & $10.2 \sim 10.6$ & $30 \sim 30.6$ & $10.2 \sim 10.6$ \\
& $L_{2}$ & $8.08 \sim 12.2$ & $23.8 \sim 33.9$ & $8.07 \sim 12.2$ \\
\hline
\end{tabular}

equals $f_{s}$, the PM is changed to $37^{\circ}$; if the control frequency is twice $f_{s}$, the PM is $43.5^{\circ}$. It should be mentioned that the PM can be improved if $a_{i}$ increases. The aforementioned design is still effective.

\section{LCL Parameters Variations}

Filter parameters may have small variations during operations. Hence, the robustness needs to be investigated. In the following, the controller parameters are the same as the above paragraph. The control parameters are kept unchanged and the filter parameters vary in a range between $\pm 50 \%$ of the nominal ones. The open-loop characteristics of (15) are analyzed. The detailed ones are shown in Table II. It can be seen that the gain of the fundamental frequency (GF), the PM and the GM all change within limited ranges. These data indicate that Types I and III have the same robustness performance for the dynamic and resonance rejection because the PMs and GMs are still over $40^{\circ}$ and $8 \mathrm{~dB}$, while Type III realizes a better steady-state response. Type II is also robust but its performance is poor.

\section{Harmonics Rejection}

Grid distortion is the main cause of current harmonics. In order to study the grid harmonics rejection performance, the Mason's gain formula method expressed in (4) is adopted. There are two forward paths from $u_{g}$ to $i_{L 2}$. In addition, the feedbacks of the capacitor voltage and the inverter-side inductor voltage have different impacts. Thus one more expression shown in (17) is needed (where, the subscripts ' $C$ C1' and ' $L 2$ ' denote capacitor voltage feedback and the inverter-side inductor voltage feedback, respectively). Then, the detailed terms of (4) for calculating the grid impact are given in (18).

$$
\left\{\begin{array}{c}
p_{P}=p_{P_{-} C 1}+p_{P_{-} L 2} \\
p_{I}=p_{I_{-} C 1}+p_{I_{-} L 2} \\
p_{D}=p_{D_{-} C 1}+p_{D_{-} L 2}
\end{array}\right.
$$




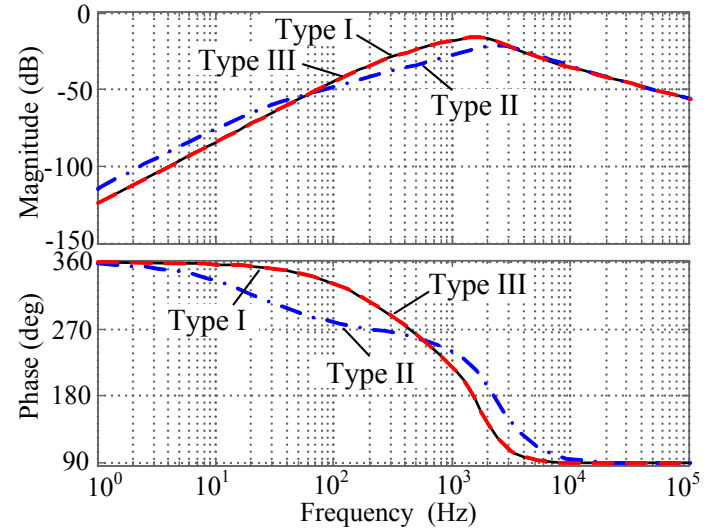

Fig. 8. Harmonics rejections of the three types.

$$
\left\{\begin{array}{l}
n=2 \quad d_{1}=k_{g} \cdot \frac{1}{L_{1} s_{1}} \cdot \frac{1}{C_{1} s} \cdot \frac{1}{L_{2} s_{2}} \quad \Delta_{1}=0 \quad d_{2}=-\frac{1}{L_{2} s} \\
\Delta_{2}=-\left[\begin{array}{l}
\frac{1}{L_{1} s} \cdot \frac{1}{C_{1} s}+\frac{y_{I}}{s}+\frac{1}{L_{1} s_{1}} \cdot\left(x_{P}+\frac{x_{I}}{s}\right)+\frac{1}{L_{1} s} \cdot\left(z_{P}+\frac{z_{I}}{s}\right) \\
+\frac{1}{L_{1} s} \cdot \frac{1}{C_{1} s} \cdot\left(p_{P_{-} C 1}+\frac{p_{I_{-} C 1}}{s}+p_{D_{-} C l} s\right)
\end{array}\right] \\
{\left[\begin{array}{l}
\frac{1}{L_{1} s} \cdot \frac{1}{C_{1} s}+\frac{1}{C_{1} s} \cdot \frac{1}{L_{2} s}+\frac{y_{I}}{s}+\frac{1}{L_{1} s} \cdot\left(x_{P}+\frac{x_{I}}{s}\right)+\frac{1}{L_{1} s} \cdot\left(z_{P}+\frac{z_{I}}{s}\right) \\
+\frac{1}{L_{1} s} \cdot \frac{1}{C_{1} s} \cdot\left(p_{P}+\frac{p_{I}}{s}+p_{D} s\right)+\frac{1}{L_{1} s_{1}} \cdot \frac{1}{C_{1} s} \cdot \frac{1}{L_{2} s} \cdot G_{c}(s) \\
+\frac{1}{L_{1} s} \cdot \frac{1}{C_{1} s} \cdot \frac{1}{L_{2} s} \cdot\left(q_{P}+\frac{q_{I}}{s}+q_{D} s\right)
\end{array}\right]} \\
\sum L_{b} L_{c}=-\left(\frac{y_{I}}{s}\right) \cdot\left(-\frac{1}{C_{1} s} \cdot \frac{1}{L_{2} s_{2}}\right)-\left(x_{P}+\frac{x_{I}}{s}\right) \cdot\left(\frac{1}{L_{1} s_{1}}\right) \cdot\left(-\frac{1}{C_{1} s} \cdot \frac{1}{L_{2} s}\right)
\end{array}\right.
$$

Then, the transfer function can be expressed as:

$$
G_{u_{g}}^{i_{2}}(s)=\frac{s \cdot\left[\begin{array}{l}
k_{g}-p_{I_{-} C l} s^{-1}-\left(1+x_{I} C_{1}+z_{I} C_{1}+p_{P_{-} C 1}\right) \\
-\left(x_{P} C_{1}+y_{I} L_{1} C_{1}+z_{P} C_{1}+p_{D_{-} C 1}\right) s-L_{1} C_{1} s^{2}
\end{array}\right]}{b_{0} s^{4}+b_{1} s^{3}+b_{2} s^{2}+b_{3} s^{1}+b_{4} s^{0}+s G_{c}(s)}
$$

The most commonly-used grid feedforward is $k_{g}=1$. Clearly, this cannot fully compensate grid distortion. Seen from (19), the grid feedforward needs to be modified to fully compensate the grid distortion. The proportional feedforward is modified:

$$
k_{g}=1+x_{I} C_{1}+z_{I} C_{1}+p_{P_{-} C 1}
$$

In addition, first-order and second-order differential compensators are required. It should be noted that the first-order differential part is produced by the choice of feedbacks. Thus this can be avoided. The derivative function is difficult to adopt.

Figure 8 shows the rejection performance when only (20) is used, and the parameters are the same as above. Types I and III have guaranteed proper rejection performance of low-frequency harmonics (for example, $-21.6 \mathrm{~dB}$ at $650 \mathrm{~Hz}$ ). Type II provides better rejection performance $(-31.5 \mathrm{~dB}$ at $650 \mathrm{~Hz})$. Generally, the feedforward function expressed in (20) is preferred because it is simple.

\section{E. Recommendations and Design Procedure}

According to the above findings, the design for the systematic control strategy can be realized. In addition, some guidelines and trade-off recommendations for choosing the feedbacks are given:

1. In order to guarantee excellent performance, Types I and III are preferred. In addition, Type III can guarantee better steady-state response of the fundamental frequency. Otherwise, if the transient performance is not considered, Type II may be suitable for current control in the synchronous rotating frame because its harmonics rejection is better.

2. The feedback combinations for all of the control types should realize the assignment of every coefficient in (6). It is to be emphasized that, for instance, because $x_{P}$ occurs in both $b_{1}$ and $b_{3}$, if the proportional feedback of the inverter-side current is chosen, one of the following $z_{P}, p_{D}, p_{I}$ or $q_{P}$ must also be chosen. In this case, if the Type I strategy is required, the value of $L_{2} p_{I}$ or $q_{P}$ must be the negative of $x_{P}$.

3 . For the purpose of involving the lowest number of sensors, for Type I, only the capacitor current or a voltage sensor is required. For Type II, there is a choice that requires only one sensor (the sampling of the capacitor or the grid-side inductor voltage). For Type III, at least two sensors are required. In addition, since the grid-side current is always measured in the control strategy shown in Fig. 4, the choice of the grid-side current feedbacks for the inner-loop control does not increase the cost.

4. In practical applications where the inverter-side current is sampled with a simple low-precision circuit for over-current protection, this current feedback may also be preferred but with the demand of a higher-precision current sensor.

5. As long as the capacitor current only consists of switching harmonics and a small amount of fundamental current its value is small. The cost of the capacitor current sensor would be quite different from the others. In addition, the sensor of grid-side inductor voltage has the same characteristic.

6. For inverters that work in both grid-connected and stand-alone modes, the feedback of the capacitor voltage may be preferred because this voltage is always sampled.

7. When the grid voltage is badly distorted, the feedbacks of the capacitor voltage and current increase the low-frequency harmonics. Then, the grid-side inductor voltage feedback which performs the same assignment is preferred. Otherwise, the grid feedforward needs be modified or harmonics resonant controllers [14], [15] are required.

As a summary of this section, the design procedure is given as follows. Firstly, depending on the practical use and the recommendations, the control type and the feedback are chosen. Secondly, the parameters in (10), (11) or (12) are determined and then the parameters for the inner feedback control are calculated. Lastly, the outer PI regulator and feedforward compensator are obtained with the use of (14) 
and (20). Note that other current regulators such as the PR controller, the repetitive controller and so on are also feasible since the resonance has been highly damped.

\section{VERIFICATIONS}

\section{A. Simulation Results}

Saber simulations in the stationary frame have been carried out. The inverter in Fig. 1 has been built. A 400V-voltage source is used as the dc-link. The grid side is a $220 \mathrm{~V} / 50 \mathrm{~Hz}$-voltage source. The feedback choices are as follows:

Type I: $\mathrm{X}=i_{C 1} \cdot z_{P}+i_{C 1} \cdot \frac{z_{I}}{S}$ with $z_{P}=16.97$ and $z_{I}=0$;

Type II: $\mathrm{X}=i_{L 1} \cdot x_{P}+u_{C 1} \cdot p_{P}+i_{L 2} \cdot q_{P}$ with $x_{P}=50.91, p_{P}=5.76$, $q_{P}=16.97$;

Type III: $\mathrm{X}=i_{C 1} \cdot z_{P}+i_{C 1} \cdot \frac{z_{I}}{s}+i_{L 2} \cdot q_{P}+i_{L 2} \cdot \frac{q_{I}}{s}$ with $z_{P}=16.97$, $z_{I}=98.70, q_{P}=0.017$ and $q_{I}=197.40$.

Note that because the frequency $\omega_{n}$ equals $\omega_{\text {res }}$ in (13), $z_{I}$ of Type I is 0 (For other cases where $\omega_{n}$ does not equal $\omega_{\text {res }}$, $z_{I}$ of Type I is no longer 0 ). In this case, this method is the same as the dual-loop current control in [7]-[9]. In addition, the capacitor voltage feedback method in [10]-[11] is yielded if $p_{D}$ with a low-pass filter to attenuate the high-frequency noises is chosen for Type I. As for Type II, the state feedback method in [13] is produced. The setup used for the simulation is just one presentation of the Type II method. The systematical structure consists of not only the existing feedback-based methods but also novel methods with different feedback choices or different pole assignment algorithms.

Figure 9 shows the steady-state waveforms of $i_{L 2}$ and the error current $\left(i_{e}=i_{\text {ref }}-i_{L 2}\right)$. No resonance phenomena occur. Currents of Types I and III are synchronous with the reference (grid voltage). However, with the Type II strategy, not only the amplitude but also the phase does not match with the reference. Type II control in the stationary frame is not appropriate. In addition, as can be seen from the error waveforms, the steady-state error of Type III is smaller. The RMS value of the error current is about $0.35 \mathrm{~A}$ which is less than $2 \%$ of the reference. This is mainly due to the grid voltage which produces a current of $0.33 \mathrm{~A}$ (the gain is $-56.5 \mathrm{~dB}$ at $50 \mathrm{~Hz}$ in Fig. 8). However, since the dc-link control can automatically adjust the amplitude during operation, there is no need to worry about the amplitude error.

Furthermore, Fig. 10 shows the waveforms when the reference suddenly changes from the rated to $10 \%$ of the rated, and then back to the rated. Clearly, the grid current can rapidly follow the reference with the Type I and Type III strategies, while the transient response of Type II is poor (about one cycle). These agree well with the analysis.

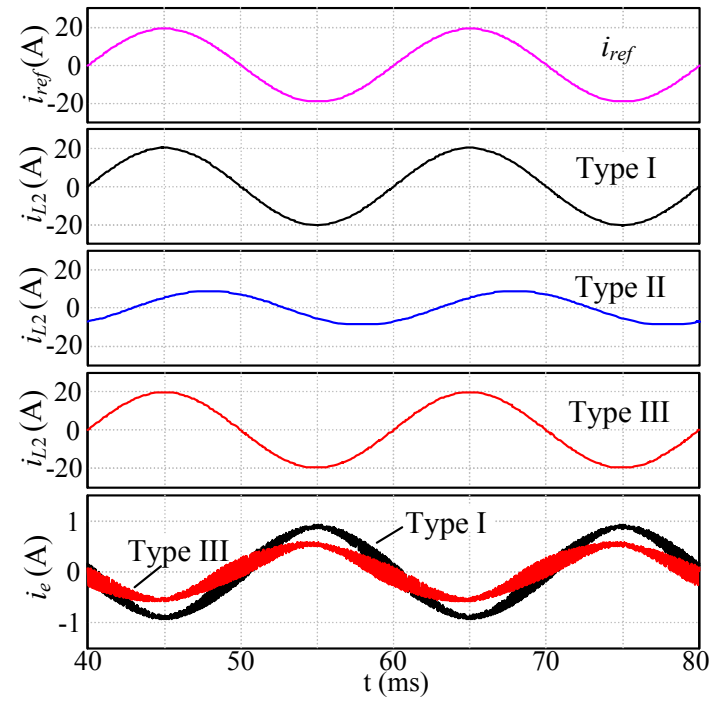

Fig. 9. Simulation results: steady-state waveforms.

\section{B. Experimental Results}

Experiments have been tested on a $5 \mathrm{~kW}$ single-phase grid-connected inverter. In order to reduce the leakage current, the inverter topology in [21] is used. The dc-link voltage is rectified by a three-phase voltage regulator and manually set to be $400 \mathrm{~V}$. The grid side is the mains ac grid $220 \mathrm{~V} / 50 \mathrm{~Hz}$. The control has been carried out with a TI TMS320F28035 DSP. The LCL-type filter parameters are: $L_{1}$ $=0.6 \mathrm{mH}, L_{2}=0.36 \mathrm{mH}, C_{1}=7 \mu \mathrm{F}$, and $f_{s}=15 \mathrm{kHz}$. For the purpose of protecting the inverter bridge, inverter-side current protection is required. Thus the sensors of the inverter-side and grid-side currents and the grid voltage are used. That is, the first, second and fourth recommendations are followed. Then, the feedback choices are as follows:

Type I: $\mathrm{X}=i_{L 1} \cdot x_{P}+i_{L 1} \cdot \frac{x_{I}}{s}+i_{L 2} \cdot q_{P}+i_{L 2} \cdot \frac{q_{I}}{s}$ with $x_{P}=18.15, x_{I}$ $=0, q_{P}=-18.15$ and $q_{I}=0$;

Type III: $\mathrm{X}=i_{L 1} \cdot x_{P}+i_{L 1} \cdot \frac{x_{I}}{s}+i_{L 2} \cdot q_{P}+i_{L 2} \cdot \frac{q_{I}}{s}$ with $x_{P}=18.15$, $x_{I}=173.21, q_{P}=-18.14$ and $q_{I}=-78.46$.

Note that the parameters are obtained by the use of the aforementioned design method. $\zeta_{0}$ for Type III is 0.01 to guarantee a robust performance when the grid fundamental frequency varies slightly.

At first, the single grid current control expressed in Fig. 3 has been tested. As indicated in equation (1), the ESRs have a slight damping effect. However, the PI parameters are restricted. The results are given in Fig. 11. For the purpose of safety, the current value is only about $30 \%$ of the rated one. The grid current is obviously resonated due to inappropriate parameters. Thus it is necessary to greatly decrease $k_{p}$ or to introduce an active damping method. However, only the second way is proper for good dynamic performance. 


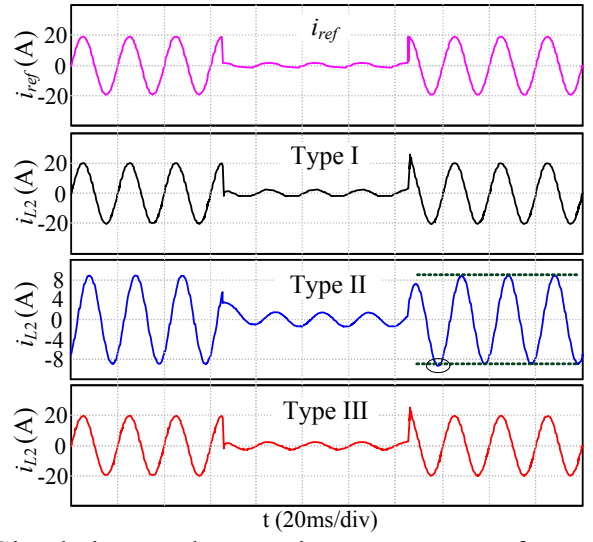

Fig. 10. Simulation results: transient current waveforms.

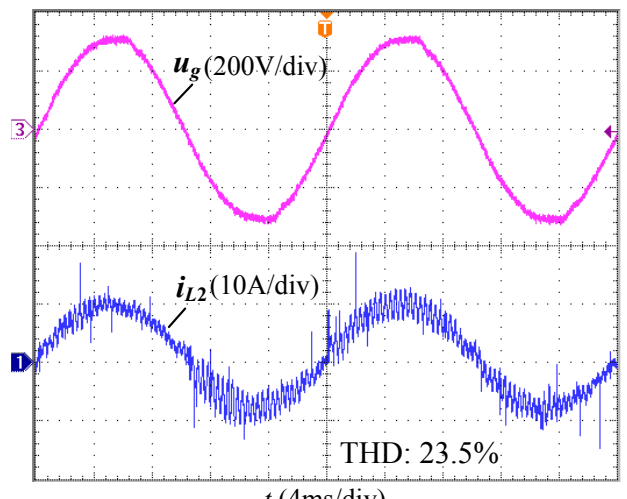

Fig. 11. Experimental waveforms with single current control.

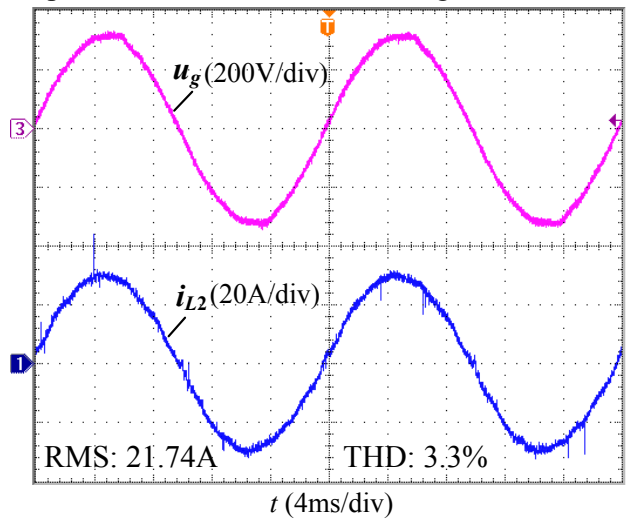

(a)

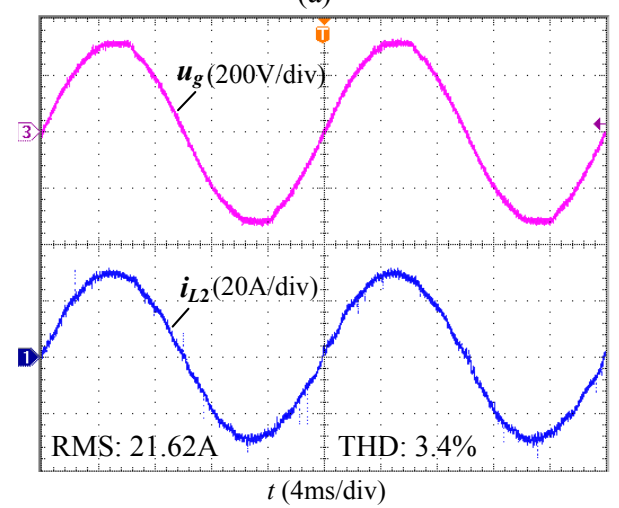

(b)

Fig. 12. Steady-state waveforms with (a) Type I and (b) Type III strategies.

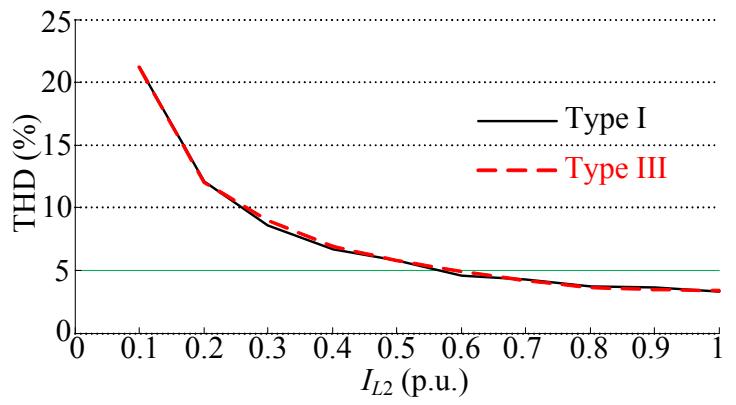

(a)

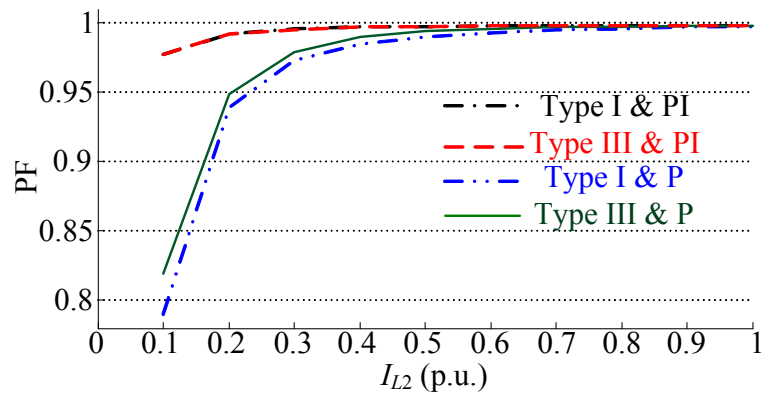

(b)

Fig. 13. Experimental results of (a) THDs and (b) PFs.

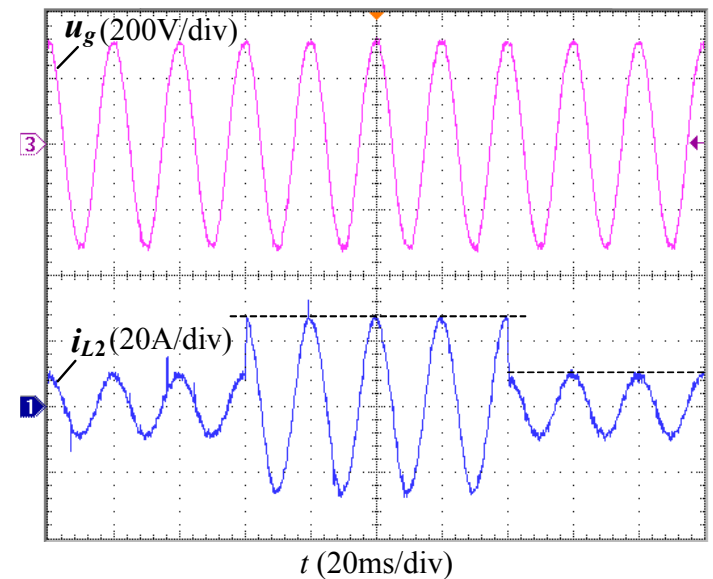

(a)

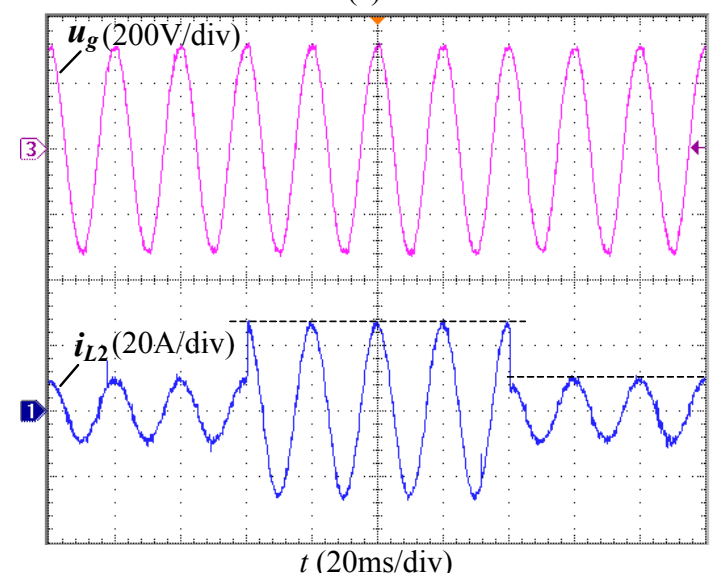

(b)

Fig. 14. Transient waveforms with Types (a) I and (b) III strategies. 
The systematic control shown in Fig. 4 is then tested. The steady-state results in the full power case are given in Fig. 12. Obviously, the harmonics are highly attenuated. In addition, Figure 13 shows detailed diagrams of the THDs and the PFs (power factors). The THDs of the two types are almost the same, as indicated in Fig. 8. With PI control, the PFs of Types I and III are also the same (0.998 at full load), as discussed in the above section. It should be noted that even at $10 \%$ of the full power case, the unit power factor has almost been maintained. In addition, the PF data with the P control have indicated that less steady-state error is guaranteed with the Type III strategy.

For the purpose of verifying the dynamic performance, experiments where the reference steps up and down have been carried out. The corresponding results are shown in Fig. 14. Clearly, Types I and III both realize good transient response. These results agree well with the theoretical and simulation analysis. The overall system control and the design strategies have been verified.

\section{CONCLUSIONS}

For grid-connected LCL-filtered inverters, a systematic control strategy has been proposed and analyzed in this paper. The contributions of this study are to further improve the control and implementation of LCL-filtered inverters, and to reveal the differences and similarities between the different methods in terms of feedback choices, resonance rejection and dynamic performance. The existing control strategies including the capacitor current feedback, the capacitor voltage feedback and the multi-state feedback methods are the typical representations of the systematical structure. Note that the basis of the weighted current control is different from the proposed one. The weighted current is the sum of the inverter-side and grid-side currents with a proper weight. In this way the zeros of the transfer function from the inverter output voltage to the weighted current are assigned to be exactly the same as the resonance poles so that the resonance phenomenon does not exist in the weighted current.

With different choices for the inner-loop feedback, different strategies are produced in this paper. The choices of sensors and control types are flexible. Among all of the control types, a novel pole-placement algorithm which simply integrates fundamental resonant control and active damping has been proposed. The performance with different strategies has been fully analyzed and verified. Based on the given guidelines and recommendations, it is easy to achieve good steady-state and dynamic performance for grid-connected inverters according to practical demands. It is hoped that this work will provide a useful reference for engineers and researchers.

It should be noted that this paper proved that a digital control delay would has an impact on stability margins and dynamic performance. The larger the ratio of the control frequency to the resonance frequency, the slighter this impact is. This ratio depends on the default parameters. For different LCL parameters, the impacts would be different. This issue will be emphasized in future work.

\section{ACKNOWLEDGMENT}

This work was supported by National Natural Science Foundation of China (51077070), Science and Technology Support Program of Jiangsu Province (BE2010188), and Funding of Jiangsu Innovation Program for Graduate Education (CXZZ12_0153, the Fundamental Research Funds for the Central Universities).

\section{REFERENCES}

[1] F. Blaabjerg, R. Teodorescu, M. Liserre, and A. Timbus, "Overview of control and grid synchronization for distributed power generation systems," IEEE Trans. Ind. Electron., Vol. 53, No. 5, pp. 1398-1409, Oct. 2006.

[2] M. Liserre, F. Blaabjerg, and S. Hansen, "Design and control of an LCL-filter-based three-phase active rectifier," IEEE Trans. Ind. Appl., Vol. 41, No. 5, pp. 1281-1291, Sep./Oct. 2005.

[3] X. Guo, X. You, X. Li, R. Hao, and D. Wang, "Design method for the LCL filters of three-phase voltage source PWM rectifiers," Journal of Power Electronics, Vol. 12, No. 4, pp. 559-566, Jul. 2012.

[4] M. Liserre, R. Teodorescu, and F. Blaabjerg, "Stability of photovoltaic and wind turbine grid-connected inverters for a large set of grid impedance values," IEEE Trans. Power Electron., Vol. 21, No. 1, pp. 263-272, Jan. 2006.

[5] J. Dannehl, C. Wessels, and F. Fuchs, "Limitations of voltage-oriented pi current control of grid-connected PWM rectifiers with LCL filters," IEEE Trans. Ind. Electron., Vol. 56, No. 2, pp. 380-388, Feb. 2009.

[6] M. Liserre, A. Dell'Aquila, and F. Blaabjerg, "Genetic algorithm-based design of the active damping for an LCL-filter three-phase active rectifier," IEEE Trans. Power Electron., Vol. 19, No. 1, pp. 76-86, Jan. 2004.

[7] A. Xu, Z. Xu, S. Xie, and M. Zou, "Study on dual-loop grid current control strategy for grid-connected inverter with an LCL-filter" in Proc. IEEE ICIEA, pp. 3200-3203, 2009.

[8] F. Liu, Y. Zhou, S. Duan, J. Yin, B. Liu, and F. Liu, "Parameter design of a two-current-loop controller used in a grid-connected inverter system with LCL filter," IEEE Trans. Power Electron., Vol. 56, No. 11, pp. 4483-4491, Nov. 2010.

[9] X. Wang, X. Ruan, S. Liu, and C. K. Tse, "Full feedforward of grid voltage for grid-connected inverter with LCL filter to suppress current distortion due to grid voltage harmonics," IEEE Trans. Power Electron., Vol. 25, No. 12, pp. 3119-3127, Dec. 2010.

[10] J. Dannehl, F. Fuchs, S. Hansen, and P. Thøgersen, "Investigation of active damping approaches for PI-based current control of grid-connected PWM converters with LCL filters," in Proc. IEEE ECCE, pp. 2998-3005, 2009.

[11] H. Kim, T. Yu, and S. Choi, "Indirect current control algorithm for utility interactive inverters in distributed generation systems," IEEE Trans. Power Electron., Vol. 23, No. 3, pp. 1342-1347, May 2008.

[12] M. Malinowski and S. Bernet, "A simple voltage sensorless 
active damping strategy for three-phase PWM converters with an LCL filter," IEEE Trans. Ind. Electron., Vol. 55, No. 4, pp. 1876-1880, Apr. 2008.

[13] J. Dannehl, F. W. Fuchs, and S. Hansen, "PI state space current control of grid-connected PWM converters with LCL filters," IEEE Trans. Power Electron., Vol. 25, No. 9, pp. 2320-2330, Sep. 2010.

[14] G. Shen, X. Zhu, J. Zhang, and D. Xu, "A new feedback method for PR current control of LCL-filter-based grid-connected inverter," IEEE Trans. Ind. Electron., Vol. 57, No. 6, pp. 2033-2041, Jun. 2010.

[15] R. Teodorescu, F. Blaabjerg, U. Borup, and M. Liserre, "A new control structure for grid-connected LCL PV inverters with zero steady-state error and selective harmonic compensation," in Proc. IEEE APEC, pp. 580-586, 2004.

[16] M. Castilla, J. Miret, J. Matas, L. Garcia de Vicuna, and J. M. Guerrero, "Control design guidelines for single-phase grid-connected photovoltaic inverters with damped resonant harmonic compensators," IEEE Trans. Ind. Electron., Vol. 56, No. 11, pp. 4492-4501, Nov. 2009.

[17] Y. Tang, P. C. Loh, P. Wang, F. H. Choo, F. Gao, and F. Blaabjerg, "Generalized design of high performance shunt active power filter with output LCL filter," IEEE Trans. Ind. Electron., Vol. 59, No. 3, pp. 1443-1452, Mar. 2012.

[18] S. J. Mason, "Feedback theory - some properties of signal flow graphs," Proceedings of the IRE, Vol. 41, No. 9, pp. 1144-1156, Sep. 1953.

[19] S. J. Mason, "Feedback theory - further properties of signal flow graphs," Proceedings of the IRE, Vol. 44, No. 7, pp. 920-926, Jul. 1956

[20] F. Golnaraghi and B. C. Kuo, Automatic control systems, John Willey\&Sons, chap. 5, 9th edn. 2009

[21] H. Xiao, S. Xie, Y. Chen, and R. Huang, "An optimized transformerless photovoltaic grid-connected inverter." IEEE Trans. Ind. Electron., Vol. 58, No.5, pp. 1887-1895, May 2011.

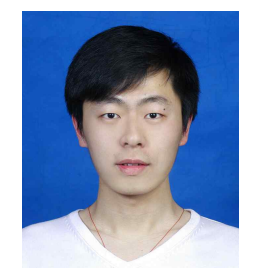

Jinming Xu was born in Xuzhou, China, in 1987. He received his B.S. in Electrical Engineering from the Nanjing University of Aeronautics and Astronautics, Nanjing, P.R. China, in 2009. From 2009 to 2011, he was a M.S. candidate in Electrical Engineering at the Nanjing University of Aeronautics and Astronautics, where he is currently working toward his Ph.D. His current research interests include grid-connected inverters and their control strategies.

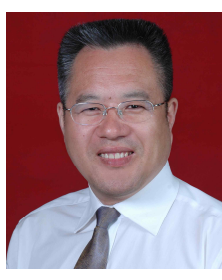

Shaojun Xie was born in Hubei, China, in 1968. He received his B.S., M.S., and Ph.D. in Electrical Engineering from the Nanjing University of Aeronautics and Astronautics (NUAA), Nanjing, P.R. China, in 1989, 1992, and 1995, respectively. In 1992, he joined the faculty of the Electrical Engineering Teaching and Research Division, NUAA, where he is currently a Professor in the College of Automation Engineering. He has authored over 50 technical papers published in many international journals and conference proceedings. His current research interests include aviation electrical power supply systems and power electronic conversion.

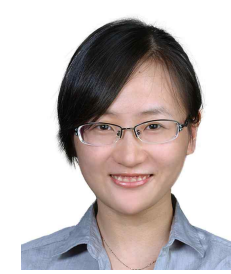

Ting Tang was born in China, in 1988. She received her B.S. in Electrical Engineering from the Nanjing University of Aeronautics and Astronautics, Nanjing, P.R. China, in 2010, where she has been a M.S. candidate since 2010. Her current research interests include photovoltaic grid-connected inverters and their control strategies. 